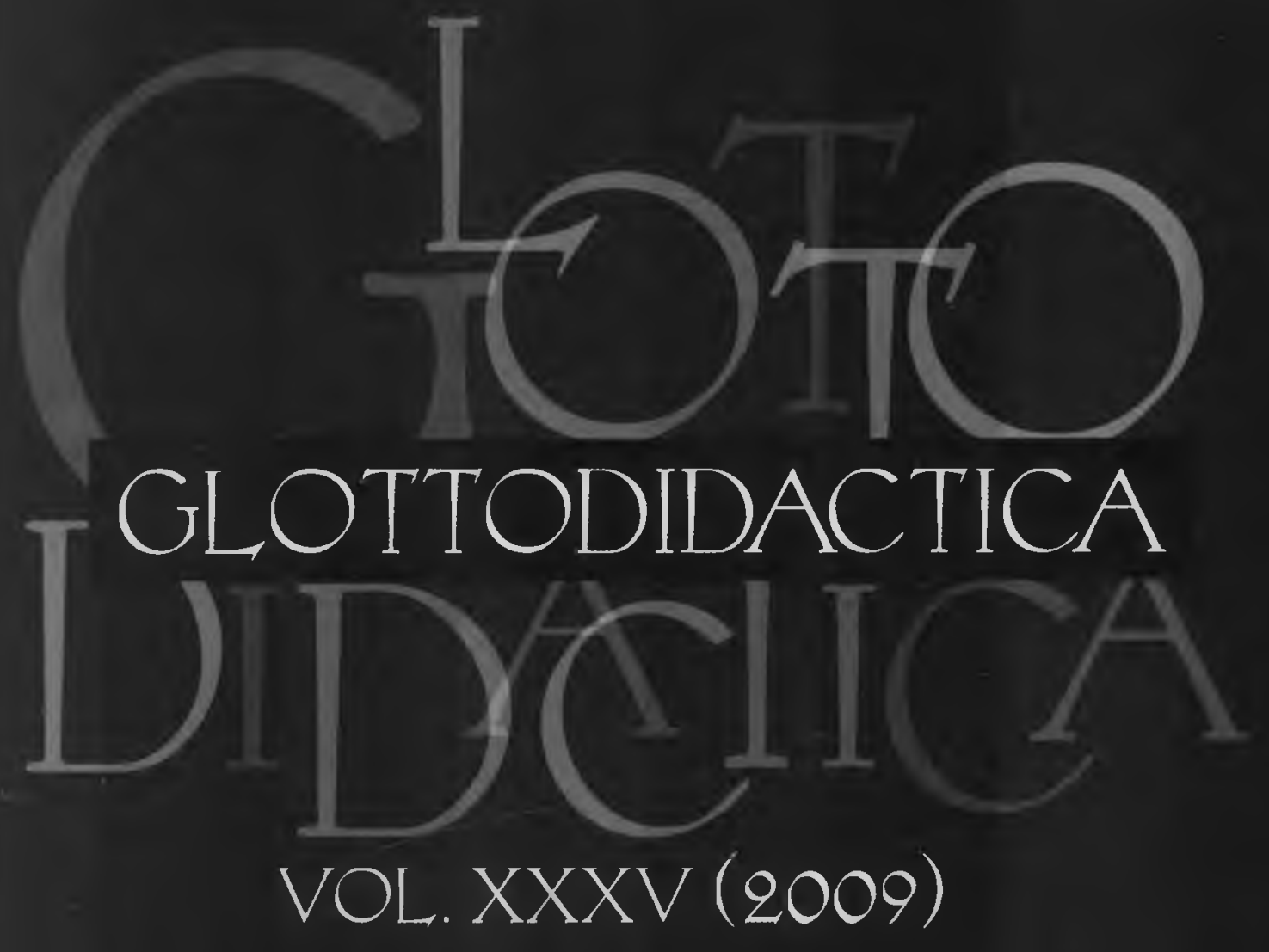

WYDAWNICTWO NAUKOWE UAM 



$$
\operatorname{cod} .42582 \wedge \frac{11}{1309}
$$

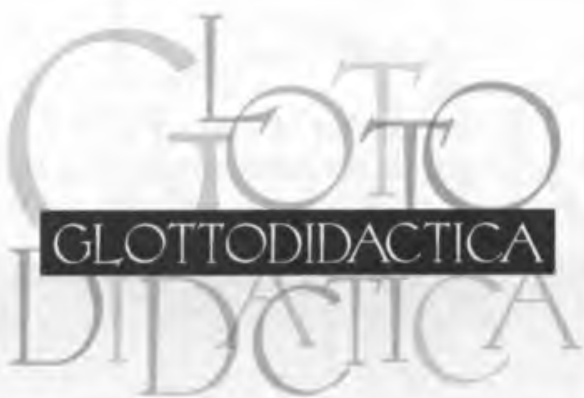




\section{GLOTTODIDACTICA \\ An International Journal of Applied Linguistics \\ Founding Editor: Prof. Dr. Ludwik Zabrocki}

Ausgabe / Issue 35

Herausgeber / Publisher

Institut für Angewandte Linguistik der Adam Mickiewicz Universität Poznań

V.i.S.d.P. Responsible person in accordance with Polish press law

Prof. Dr. Waldemar Pfeiffer (Honorary Editorship)

Redaktion (Leitung) / Editorial Head

Prof. Dr. Barbara Skowronek

Redaktionelle Mitarbeit / Editorial support

Dr. Luiza Ciepielewska-Kaczmarek, Dr. Monika Kowalonek-Janczarek

Redaktionsbeirat Editorial / Advisory Board

Prof. Dr. Jerzy Bańczerowski, Prof. Dr. Anna Cieślicka, Prof. Dr. Józef Darski,

Prof. Dr. Jacek Fisiak, Prof. Dr. Antoni Markunas, Prof. Dr. Kazimiera Myczko,

Prof. Dr. Stanisław Puppel, Prof. Dr. Christoph Schatte, Prof. Dr. Teresa Tomaszkiewicz, Prof. Dr. Weronika Wilczyńska, Prof. Dr. Stephan Wolting, Prof. Dr. Władysław Woźniewicz

Wissenschaftlicher Beirat / Academic Advisory Board

Prof. Dr. Rupprecht S. Baur (Duisburg/Essen), Prof. Dr. Dmitrij Dobrovolskij (Moskau/Wien), Prof. Dr. Franciszek Grucza (Warszawa), Prof. Dr. Wolfgang Herrlitz (Utrecht),

Prof. Dr. Hanna Komorowska (Warszawa), Prof. Dr. Bogdan Kovtyk (Halle),

Prof. Dr. Frank Königs (Marburg/L.), Prof. Dr. Roman Lewicki (Wroclaw),

Prof. Dr. Clare Mar-Molinero (Southhempton), Prof. Dr. Paweł Mecner (Szczecin),

Prof. Dr. Bernd Müller-Jacquier (Bayreuth), Prof. Dr. David S. Singleton (Dublin), Prof. Dr. Marian Szczodrowski (Gdańsk), Prof. Dr. Iwar Werlen (Bern), Prof. Dr. Jerzy Żmudzki (Lublin) 
UNIWERSYTET IM. ADAMA MICKIEWICZA W POZNANIU

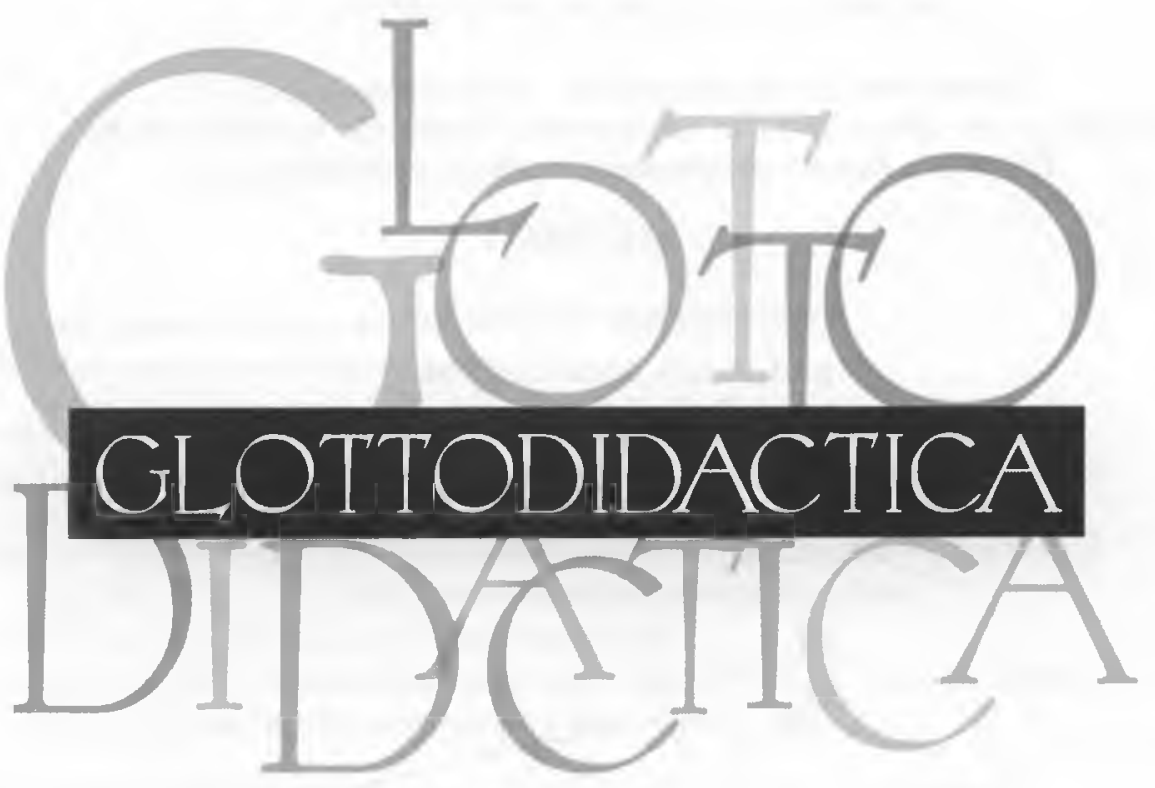

VOLUME XXXV

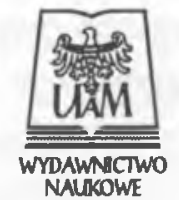

POZNAŃ 2009 


$$
\begin{gathered}
\text { Adres redakcji } \\
\text { Instytut Lingwistyki Stosowanej UAM } \\
\text { ul. } 28 \text { Czerwca } 1956 \text { r. nr } 198 \\
\text { 61-485 Poznań, Poland } \\
\text { tel. }+48618292925 \text {, tel./ fax }+48618292926
\end{gathered}
$$

Editor: Prof. Barbara Skowronek barbaras@amu.edu.pl Assistants to the Editor: Dr Luiza Ciepielewska-Kaczmarek luizac@poczta.fm

Dr Monika Kowalonek-Janczarek monika.kowalonek@wp.pl

$$
\begin{gathered}
\text { Weryfikacja językowa tekstów } \\
\text { prof. dr hab. Anna Cieślicka } \\
\text { dr Britta Stöckmann }
\end{gathered}
$$

Publikacja dofinansowana przez

Instytut Lingwistyki Stosowanej UAM

\section{POZNANI}

(C) Wydawnictwo Naukowe UAM, Poznań 2009

Wydano na podstawie maszynopisu gwarantowan go

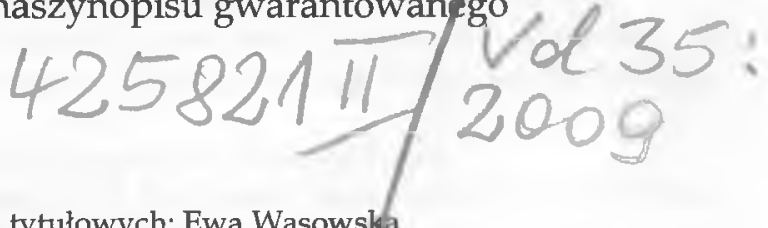

Projekt okladki i stron tytułowych: Ewa Wąsowska

Redaktor prowadzący: Anna Rąbalska

Formatowanie: Eugeniusz Strykowski

\section{ISBN 978-83-232-2083-1 ISSN 0072-4769}

WYDAWNICTWO NAUKOWE UNIWERSYTETU IM. ADAMA MICKIEWICZA

61-734 Poznań, ul. F. Nowowiejskiego 55, tel. 06182939 85, fax 0618293980 e-mail: press@amu.edu.pl www.press.amu.edu.pl

Ark. wyd. 17,00 . Ark. druk. 15,50

ZAKŁAD GRAFICZNY UAM, POZNAŃ, UL. H. WIENIAWSKIEGO 1

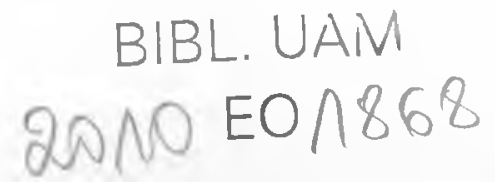




\section{CONTENTS}

\section{ARTICLES}

Basic and Referential Disciplines

Grundlagen- und Referenzwissenschaften

MARIAN SZCZODROWSKI, Wesen, Arten und Wirkungen der fremdsprachlichen Steuerung ...

GRAŻYNA LEWICKA, Zu einigen Problemen des Verstehens in der konstruktivistisch orientier-

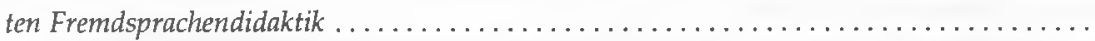

REINHOLD UTRI, Bilinguale Erziehung - neue Herausforderungen und Lösungen aus linguisti-

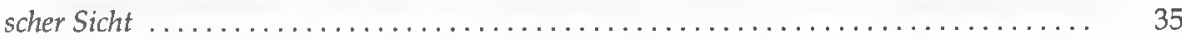

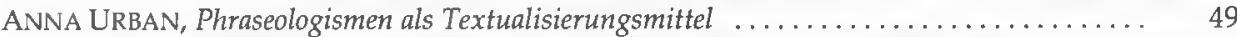

NATASCHA JOURDY, НарратиВная структура художественного текста: свободнокосвенный дискурс как категория лингвистики и нарратологии ................

\section{Methodology in Glottodidactics}

Fremdspracheninethodik

ZOFIA CHLOPEK, The relationship between language learning experience, motivation and some

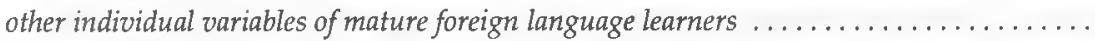

MALGORZATA CZARNECKA, Die Entwicklung interner grammatischer Regeln im gesteuerten

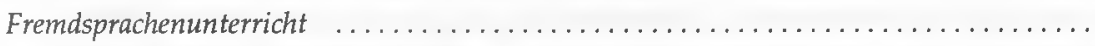

BRIGITA KOSEVSKI PULJIĆ, Der Enwerb der Schreibkompetenz im Deutsch als Fremdsprache -

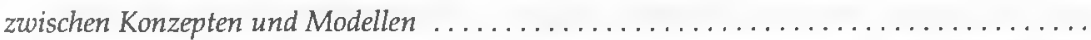

AGNIESZKA MAC, Einige Überlegungen zur Wortbildungslehre im fremdsprachlichen

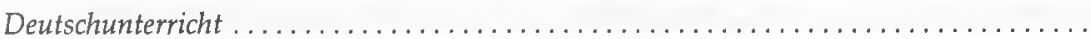

SYlWIA ADAMCZAK-KRYSZTOFOWICZ, ANTJE STORK, Podcasts im glottodidaktischen Medienverbund. Versuch einer vergleichenden Analyse ......................

MARIUSZ WALORCZYK, Optimierung der ZD-Prüfungsvorbereitung in Bezug auf die Aufgabenformen

\section{Language Policy and Intercultural Studies}

Sprachenpolitik und Interkulturelle Studien

MONIKa KOWALONEK-JANCZAREK, Die Nachbarsprache Polnisch aus einer sprachpolitischen und soziolinguistischen Perspektive im Kontext der Mehrsprachigkeit in Europa .........

NINO LOLADZE, Interkulturelles Lernen als Schlüsselqualifikation im Zeitalter der Globalisierung im Kontext des Deutschunterrichts in Georgien 


\section{RESEARCH REPORTS}

DANUTA WIŚNIEWSKA, EFL Teachers' Perception and Practice of Action Research .......... 163

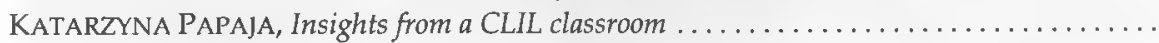

MAGDAlENA KOPER, MAGDALENA DUdZIŃSKA, Europäisierung der Sprachenpolitik im Bereich des Kulturtourismus nach dem EU-Beitritt Polens am Beispiel der Stadt Gdansk ....

\section{BOOK REVIEWS}

BARBARA SKOWRONEK, Marian Szczodrowski: Fremdsprachliche Lehr-Lern-Vorgange im kodematischen Blickfeld. Wydawnictwo Uniwersytetu Gdańskiego, Gdańsk - Sopot 2009,225 S. . . . . . . . . . . . . . . . . . . . . . . . . . . . . . .

JOANNA KIC-DRGAS, Sambor Grucza: Lingwistyka języków specjalistycznych. Wydawnictwo Euro-Edukacja, Warszawa 2008, 256 S.

ANNA PIECZYNSKA-SUlIK, Hans-Jörg Schwenk: Präfigierung im Polnischen und ihre Entsprechung im Deutschen. $\mathrm{Zu}$ Theorie von und lexikographischem Umgang mit Aspekt und Aktionsart. Reihe: Języki - Kultury - Teksty - Wiedza. Band 8, Wydawnictwo EuroEdukacja, Warszawa 2007, $301 \mathrm{~S}$.

BARBARA SKOWRONEK, Magdalena Olpińska: Nauczanie dwujęzyczne w świetle badań $i$ koncepcji glottodydaktycznych. Seria: Języki - kultury - teksty - wiedza. Franciszek Grucza, Jerzy Lukszyn (Hg.). Wydawnictwo Euro-Edukacja, Warszawa 2008, 247 S. . . . . . .

REINHOLD UTRI, Marc Bielefeld: We spe@k Deutsch... aber verstehen nur Bahnhof. Unterwegs im Dschungel unserer Sprache. Wilhelm Heyne Verlag, München, 2008, 286 S. .

GABRIELA GORĄCA, Sambor Grucza: Od lingwistyki tekstu do lingwistyki tekstu specjalistycznego. Seria: Języki - kultury - teksty - wiedza. Franciszek Grucza, Jerzy Lukszyn (Hg.). Wydawnictwo Euro-Edukacja, Warszawa 2007, 218 S.

KATARZYNA KONSEK, Jörg Roche: Handbuch Mediendidaktik. Hueber Verlag, Ismaning $2008,176 \mathrm{~S}$.

ANNA URBAN, Christiane Hümmer: Synonyme bei phraseologischen Einheiten. Eine korpusbasierte Untersuchung. Peter Lang, Frankfurt am Main 2009, 357 S. . . . . . . . .

SYLWIA ADAMCZAK-KRYSZTOFOWICZ, Kazimiera Myczko, Barbara Skowronek, Władyslaw Zabrocki (red.): Perspektywy glottodydaktyki $i$ jezykoznawstwa. Tom jubileuszowy $z$ okazji 70. urodzin Profesora Waldemara Pfeiffera. Wydawnictwo Naukowe UAM, Poznań 2008, 499 S. . . . . . . . . . ..............................

MONIKA KOWALONEK-JANCZAREK, Boris Blahak, Clemens Piber (Hg.): Deutsch als fachbezogene Fremdsprache in Grenzregionen. Ekonóm, Bratislava 2008, 322 S. . . . . . . .

LUIZA CIEPIELEWSKA-KACZMAREK, Sambor Grucza (Hg.): W kręgu teorii i praktyki lingwistycznej. Księga jubileuszowa poświęcona Profesorowi Jerzemu Lukszynowi z okazji 70. rocznicy urodzin. Wydawnictwo Uniwersytetu Warszawskiego, Warszawa 2007, 367 S. . . . . . . . .

AGNIESZKA PAWŁOWSKA, Kultury i języki poznawać - uczyć się - nauczać / Kulturen und Sprachen verstehen - lemen - lehren. Pod redakcją / Herausgegeben von Anna Jaroszewska, Marta Torenc. Uniwersytet Warszawski, Instytut Germanistyki, Warsza-

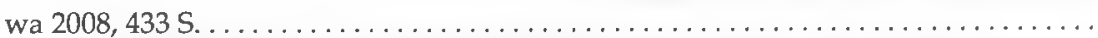

BEATA MikOŁAJCZYK, Konrad Ehlich, Dorothee Heller (Hg.): Die Wissenschaft und ihre Sprachen. 2006. Peter Lang Verlag, Bern / Berlin / Bruselles / Frankfurt a.M. / New York / Oxford / Wien. (= Linguistic Insights. Studies in Language and Communi-

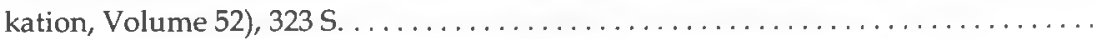




\section{REPORTS}

LUIZA CIEPIELEWSKA-KACZMAREK, Lesen und Leseverstehen in der DaF-Didaktik

MAGDAlena JUREWICZ, PAWEE RYBSZLEGER, Bericht über die Internationale wissenschaftliche Konferenz des Verbandes Polnischer Germanisten (VPG) ..........

AGNIESZKA PAWŁOWSKA, Bericht über die internationale Konferenz Reflexion als Schlüsselphänomen der gegenwärtigen Fremdsprachendidaktik

GRAŻYNA ZENDEROWSKA-KORPUS, Das linguistisch-hochschuldidaktische Kolloquium „Fachsprachenpropädeutik im Germanistikstudium" 



\title{
Einige Überlegungen zur Wortbildungslehre im fremdsprachlichen Deutschunterricht
}

\author{
Some reflections on a word formation \\ in teaching German as a foreign language
}

ABSTRACT. The goal of this article is to expatiate on the important function of the morphological structures in foreign language teaching. Firstly, I will try to answer the question about morphology's position in foreign language teaching. Furthermore, I will focus on the difficulties arising with implementing teaching methods of word formation rules. The third part deals with the effects of morphology in the didactical field with regard to teaching German as a foreign language.

Keywords: teaching German as a foreign language, textbooks, word formation.

Wortbildung stellt die wichtigste Möglichkeit der Wortschatzerweiterung im Deutschen dar und spielt somit eine bedeutende Rolle im Sprachgebrauch und im Sprachsystem. Will man sich einen Eindruck darüber verschaffen, wie hoch der Anteil von Wortbildungen an der Sprache ist, reicht es bereits aus, einen Zeitungsausschnitt, den Abschnitt aus einem Buch, im Prinzip eine beliebige Textpassage, so z.B. eine Seite aus einem DaFLehrwerk (mit verschiedenen Übungen zu unterschiedlichen Fertigkeiten ${ }^{1}$ ) unter diesem Gesichtspunkt zu sondieren und zu analysieren. Anhand der Frequenz der dort vorkommenden Wortbildungsprodukte kann man schlussfolgern, dass und in welchem Ausmaß Wortbildungsprozesse als

${ }^{1}$ Siehe z.B. Team Deutsch LB2, S. 17, 25, 60, 62. 
zentraler Bestandteil des Fremdsprachenunterrichts zu betrachten sind, denen man "[...] gleichberechtigt neben Syntax, Formenbildung, Wortschatz, Phonetik usw. [...]" (Storch/Storch-Luche 1979: 11) eine analoge, hohe Aufmerksamkeit im Unterrichtsgeschehen widmen sollte. Die Lektüre der DaF-Lehrwerke lässt aber nicht erkennen, dass die Wortbildung zu den grundlegenden Elementen des Fremdsprachenunterrichts gehört. Zwar werden in den Lehrwerken Übungen zur Wortbildung angeboten (in manchen sind sie häufiger, in anderen seltener anzutreffen, wenn man vergleichbare Lehrwerke, d.h. bezogen auf gleiches Niveau und gleichen Schultyp, ${ }^{2}$ in den Blick nimmt), aber eine regelmäßige und systematische Vermittlung von Wortbildungsregeln ist kaum zu finden. Es sei denn, unzureichende Erklärungen von Wortbildungsregeln werden vorgestellt, die alles andere als optimal bezeichnet werden können und viel zu wünschen übrig lassen.

Das Ziel dieses Beitrags ist es, auf die wichtige Rolle der Wortbildungslehre im fremdsprachlichen Deutschunterricht hinzuweisen. Die folgenden Ausführungen zum Thema erheben keinen Anspruch auf Vollständigkeit. Das skizierte Bild will indessen ausgewählte Probleme aufzeigen, für die weitere komplexere Untersuchungen erforderlich sind.

\section{WARUM WORTBILDUNG IM FREMDSPRACHENUNTERRICHT?}

Der Erwerb einer fremden Sprache ist ein kreativer und konstruktiver Prozess. Der Lernende speichert (in den meisten Fällen) nicht fertige Sätze und Ausdrücke, die er "[...] bei Bedarf einfach abruft, sondern er lernt, komplexe Inhalte mit Hilfe der ihm zur Verfügung stehenden sprachlichen Regularitäten und lexikalischen bzw. idiomatischen Inhalte auszudrücken und zu verstehen" (Storch 1979a: 2). Das betrifft alle Bereiche des Sprachenlernens, darunter auch die Wortbildung. Wenn man den Lernenden im Fremdsprachenunterricht zu vermitteln vermag, wie lexikalische Einheiten regulativ gebildet sind bzw. werden, so ist ihnen damit - laut Storchs emphatischer Betonung zufolge - „[...] ein nicht hoch genug einzuschätzendes, da außerordentlich ökonomisches Instrument zur Wortschatzerweiterung an die Hand gegeben“ (Storch 1979a: 3). ${ }^{3}$ Wird man sich dieser Einschätzung in

${ }^{2}$ Wenn man z.B. Lehrwerke für das polnische Gymnasium vergleicht, gibt es in Team Deutsch recht viele Übungen zur Wortbildung im Vergleich zu Pingpong Neu oder Geni@l.

${ }^{3}$ Es soll an dieser Stelle ergänzt werden, dass es nicht reicht, nur die Regularitäten der Wortbildung zu vermitteln. Sie müssen um die Restriktionen und Irregularitäten ergänzt wer- 
ihrer ganzen Konsequenz bewusst, so bedarf es keinerlei zusätzlicher Motivation dafür, sich im Fremdsprachenunterricht intensiv mit Wortbildung auseinanderzusetzen (vgl. Storch 1979a: 3).

Saxer (1991: 55f.) weist auf drei Dimensionen der Wortbildung hin, die die Vermittlung von diversen sprachlichen Fertigkeiten beeinflussen und deshalb im Fremdsprachenunterricht als Lerngegenstand auch keineswegs vernachlässigt werden sollten. Dazu gehören:

A. Grammatische Dimension: Sie umfasst die formale Veränderung eines einfachen Wortstammes bzw. Wortes durch Zusammensetzung oder Derivation mit dem Ziel, eine neue Bedeutung zu schaffen. „Das grundlegende Prinzip der Wortbildung aus (satz-) grammatischer Perspektive ist die Kombinatorik: Wörter/Wortstämme/Silben werden miteinander kombiniert" (Saxer 1991: 55f.).

B. Semantische Dimension: Der Bereich der Bedeutungen bildet den Kern der Wortbildung. Da die durch formale Veränderungen geschaffenen, neuen Bedeutungen untrennbar von den jeweiligen situativen Zusammenhängen und den sprachlichen Intentionen der beteiligten Personen abhängen, ist der Bereich der Bedeutungen unbegrenzt und man kann daher , [...] nicht von eindeutigen Strukturen ausgehen wie bei der Satzgrammatik" (Saxer 1991: 56). Die Lernenden müssen daher von den möglichen allgemeinen Bedeutungszusammenhängen in Kenntnis gesetzt werden, damit sie mit der Bedeutung von Wortbildungsprodukten, die für sie neu sind, umgehen können (vgl. Saxer 1991: 56). Nur dann kann die Wortbildung als Hilfe beim Lernen betrachtet werden.

C. Textuelle (textgrammatische) Dimension: Wortbildungen sind auch wichtig bei der eigenen Produktion von Texten: dank Ableitungen lassen sich z.B. Wiederholungen desselben Wortes vermeiden, oder etwas vorher Berichtetes oder Beschriebenes kann in einem Wort zusammengefasst werden. ${ }^{4}$

Wie aus dem bis hierher Dargelegten ersichtlich wird, sollen Wortbildungsprozesse aufgrund ihrer syntaktischen, semantischen und textuellen Potenziale in engem Zusammenhang mit verschiedenen Lerngegenständen im fremdsprachlichen Deutschunterricht behandelt werden. Die kurz beschriebenen Dimensionen liefern Anhaltspunkte dafür, dass sich die Kenntnisse von der Wortbildungslehre und ihren Produkten beim Deutschlernen

den, damit die Lernenden ein vollständiges Bild über die Wortbildungslehre bekommen. Sie sollen jedoch im Schatten der Regularitäten stehen. Nähere Erklärungen dazu, siehe Storch 1979a: $2 f$.

${ }^{4}$ Näheres dazu siehe Saxer 1991: 56. 
als behilflich erweisen können, deshalb systematischer Vermittlung bedürfen und sich in den Übungen zu allen Fertigkeiten widerspiegeln sollten. Dafür finden sich leider jedoch nur ungenügende Belegstellen in den heutigen Lehrwerken. Zwar gibt es Aufgaben zur Grammatik- oder Wortschatzvermittlung, in denen manches erklärt oder geübt wird, aber es fehlt meistens an Vorschlägen für einen durchdachten Wortbildungsunterricht. Es gibt auch kaum Aufgaben, etwa zum Leseverstehen oder Schreiben, in denen gezielt auf die Funktion der Wortbildung für das Training dieser Fertigkeiten hingewiesen würde.

Die Wortbildung lenkt überdies die Aufmerksamkeit der Sprachverwender und Lerner auf die komplizierten Wechselbeziehungen zwischen sprachlicher und außersprachlicher Wirklichkeit. Dazu Wilss:

Sie öffnet dem kritischen Sprachbenutzer die Augen für die Abbildfunktion der Sprache; sie lässt ihn gleichsam auf eine ihn mehr oder minder intensiv betreffende Weise teilhaben an sprachlichen Entwicklungen, die im Rahmen gesamtgesellschaftlicher Veränderungen vor sich gehen und Rückschlüsse darüber zulassen, in welchem Umfang die Sprachverwendung heute umweltabhängig geworden ist, wie sehr heute sprachliche Fremdbestimmtheit über sprachliche Selbsterfahrung dominiert. Wer heute die deutsche Tages- und Wochenpresse liest, empfängt das Bild eines sprachlichen Szenarios, das die professionellen Erzeuger, Verwalter und Vertreter auf vorgespurten Bahnen täglich neu zusammensetzen (Wilss 1986: 225).

Nach Wilss (1986: 225) suggeriert die Wortbildung eine empirische Hypothese über das sprachliche Verhalten der Sprachbenutzer in vielen unterschiedlichen kommunikativen Situationen. So würden in der Wortbildung neue kommunikative Partnerbeziehungen, Partnereinschätzungen und Rollenverhältnisse sichtbar, „[...] in denen die Wortbildung wie ein festes Geländer wirkt, an dem man sich nahezu blindlings von Kommunikationssituation zu Kommunikationssituation weiterhangeln kann" (Wills 1986: 225).

Nur die oben angesprochenen Funktionen der Wortbildung erlauben es, auf ihre wichtige Rolle im Fremdsprachenunterricht zu schließen. Somit stimme ich Wilss zu, wenn er behauptet, dass die produktive und rezeptive Beherrschung von Wortbildungserscheinungen und den ihnen zugrunde liegenden Wortbildungstypen und -mustern ein wesentlicher Teil der im Laufe des Spracherwerbsprozesses zu erlernenden kommunikativen Kompetenz ist (Wilss 1986: 226). Er weist auch darauf hin, dass unter kommunikativer Kompetenz nicht zuletzt zu verstehen ist, dass ein Sprachbenutzer die Normen und Handlungsvorschriften alltagssprachlicher wie auch fachsprachlicher Kommunikation kennt (jedenfalls in bestimmtem Umfang). Darüber hinaus muss ein Sprachbenutzer wissen, wie er das lexikalische und syntaktische 
Ausdruckspotenzial einer Sprache zu aktualisieren hat. Er muss also Kenntnis darüber besitzen, „[...] wie er sich Wortbildungserscheinungen aneignet, wie er sie speichert, wie er sie mental (kognitiv-assoziativ) ordnet und wie er sie effektiv anwendet" (Wills 1986: 226f.). Er muss imstande sein, seine Lernstrategien zu entwickeln, um Denk- und Handlungsstrukturen aufzubauen, „[...] in denen kognitive, kommunikative, intersubjektive, soziale und lebensweltliche Faktoren integrativ zusammenwirken und verständigungsorientierte Kommunikation ermöglichen" (Wills 1986: 226f.).

Bei diesen, oben angeführten Handlungen, die sich auf die Wortbildungslehre im Fremdsprachenunterricht beziehen, sollen die Lehrkraft und das Lehrwerk dem Lernenden zur Seite stehen. Storch befürchtet aber, dass es oftmals gerade an fehlenden Wortbildungskenntnissen der Lehrkräfte (ganz zu schweigen vom Lehrwerk) liege, weshalb sie nicht imstande seien, die Wortbildung in den Unterricht mit einzubeziehen (Storch 1979a: 3). ${ }^{5}$ Weiter betont er, dass es fast unmöglich sein dürfte, ohne Einsichten in bestimmte theoretische Grundlagen der Wortbildungslehre vernünftige Lernziele und effektive Lehrmethoden für den Wortbildungsunterricht zu erarbeiten (Storch 1979a: 6). ${ }^{6}$ Hinzu kommt die Tatsache, dass die Wortbildung in den meisten Lehrwerken zu wenig (in getrennten Kapiteln, Abschnitten, Übungen) thematisiert wird. Am häufigsten finden sich Aufgaben, in denen bestimmte Regularitäten zwar eingeübt werden, eine nähere Erläuterung hingegen meistens bei der Lehrkraft liegt, von der es abhängt, in welchem Maße die Lernenden für die jeweilige Erscheinung sensibilisiert werden.

Dieser Abriss der Vorteile, die sich aus der Auseinandersetzung mit der Wortbildung in verschiedenen Bereichen des Fremdsprachenunterrichts ergeben, soll verdeutlichen, dass und inwiefern es sich lohnt, das Thema aufzugreifen, auch wenn das mit bestimmten Anstrengungen - meistens seitens der Lehrkräfte - einhergeht. Aber auch sie können davon profitieren, und zwar, wenn sich bei ihren Lernenden sichtbare Fortschritte beim Lernen einstellen, die sonst nicht zu erwarten sind.

${ }^{5}$ Für die Wortbildung gibt es im Rahmen des Germanistikstudiums in Polen wenig Platz. Aligemeines Wissen darüber wird in den Vorlesungen oder Seminaren zur beschreibenden Grammatik des Deutschen vermittelt, und für die unterrichtsmethodische Umsetzung der Wortbildungslehre werden die zukünftigen Deutschlehrer nur selten und wenn liberhaupt, dann in geringerem Maße am Rande bei der Wortschatzvermittlung im Methodikseminar sensibilisiert. Die Literatur für die Methodik und Didaktik des Deutschunterrichts hat in diesem Bereich auch nicht viel zu bieten, man findet zu diesem Thema lediglich ein kleines Unterkapitel bei Bohn (2000: 51-55); Storch (1999: 90-104); Löschmann (1993: 65) und Bimmel/ Rampillon (2000: 71) gehen auf das Thema bei den Lernstrategien ein, um nur einige der wenigen Beispiele in der Literatur zu nennen.

${ }^{6}$ Genauer dazu siehe Storch 1979a: 6ff. 


\section{SCHWIERIGKEITEN BEI DER UNTERRICHTSMETHODISCHEN UMSETZUNG DER WORTBILDUNGSLEHRE IM FREMDSPRACHENUNTERRICHT}

Obwohl die Wortbildung im fremdsprachlichen Deutschunterricht auf den ersten Blick bei der Wortschatzvermittlung und beim Wortschatzlernen hilfreich zu sein scheint, ist sie unter dem Gesichtspunkt von Sprachlehrund Lernanforderungen ein schwieriges Thema. Cieszkowski (1993: 19) benennt als Ursachen „[...] die Schwierigkeiten einer linguistischen Darste]lung und einer daraus resultierenden unterrichtsmethodischen Umsetzung $[\ldots]^{\prime \prime}$ :

So sind z.B. nicht nur die Fragen kategorieller Zuordnung von Wortbildungsarten zu entsprechenden Wortbildungsmodellen eindeutig entschieden, so herrscht auch wenig Klarheit über den Stellenwert der Wortbildung im System der deutschen Gegenwartssprache wie überhaupt über ihre Bedeutung für den Fremdsprachenunterricht; viele theoretische Erörterungen sind leider bisher nicht oder nur in unzureichendem Maße für den Sprachunterricht - und insbesondere für den FSU - nutzbar gemacht worden, was weitgehend eine effektive methodische Vermittlung erschwert und die zu erreichende Sprachkompetenz des Lernenden entscheidend beeinträchtigen kann (Cieszkowski 1993: 19).

Storch (1979b: 3f.; 1999: 92f.) geht dagegen konkret auf Schwierigkeiten ein, auf die die Fremdsprachenlernenden stoßen können, wenn sie sich im Unterricht mit der Wortbildung auseinandersetzen. Dabei werden von ihm die sich aus der Lernpsychologie ergebenden und psycholinguistisch bedingten Faktoren, d.h. die psychischen Faktoren jedes Lernenden, nicht berücksichtigt und nur Probleme linguistischer Art in den Blick genommen.

Eines der wichtigsten Probleme, das sich bei den Lernenden einstellen kann, ist Storch zufolge (1999: 92), dass sie als Nicht-Muttersprachler nur schwer zwischen motivierten und teil- bzw. unmotivierten Wortbildungen unterscheiden können. „Selbst im Kontext dürfte oft nicht zu erkennen sein, dass die Bedeutung von Jungfrau und besitzen, anders als bei Rotwein und besteigen, nicht auf die Basiskonstituenten zurückgeführt werden kann" (Storch 1999: 92). Aber auch motivierte Wortbildungen haben eigene sprachliche Feinheiten: „[...] Raucher hat oft den Aspekt 'gewohnheitsmäßig'; und nicht jede große Stadt ist auch eine Großstadt" (Storch 1999: 92). ${ }^{7}$

Schwieriger sollen nach ihm für die Lernenden Wortbildungen sein, die mit Hilfe von Wortbildungsaffixen gebildet werden, als solche, die nur aus lexikalischen Konstituenten bestehen. Bei Wortbildungen wie z.B. benachrichtigen oder Lieblosigkeit müssen die Affixfunktionen und die syntaktisch-

${ }^{7}$ Siehe dazu auch Cieszkowski 1993: 21. 
semantischen Strukturzusammenhänge geklärt werden, damit ihre Bedeutung erschlossen werden kann. Bei Wortbildungen mit lexikalischen Konstituenten wie z.B. Haustür, hellrot (sofern sie bekannt sind) können sie im Kontext erfahrungsgemäß relativ leicht und oft ohne zusätzliche Erklärung verstanden werden (vgl. Storch 1979b: 3).

Die nächste Schwierigkeit sieht er darin, dass Wortbildungsmittel manchmal in sehr unterschiedlichen Funktionen und manchmal umgekehrt in derselben Funktion vorkommen können. Hier liegt eine Mischung aus regelhaften und nicht regelhaften Erscheinungen vor (Storch 1999: 92). Er betont, dass polyfunktionale Wortbildungsmittel wohl schwerer zu verarbeiten sind als solche, die nur eine Funktion haben, z.B. die verschiedenen Funktionen des Verbpräfixes ver- und das monofunktionale Adjektivsuffix los (Storch 1979b: 4). Ähnlich sind Wortbildungsmuster, die sich auf mehrere Affixe derselben Funktion verteilen, wohl schwieriger als solche, die nur ein Affix umfassen, z.B. etwas befeuchten, ermöglichen, verdunkeln (deadjektivische Verben der bewirkten Zustandsveränderung) und ungenau, unfein, unschön usw. Die Lernenden können aber auch Probleme bei Affixen wie $a$-, in- und entlehnter Basis haben, weil sie die Lehnwörter nicht kenwen können, z.B. amusisch, intolerant (Storch 1979b: 3f.).

Storch (1979b: 4) behauptet des weiteren, dass nur eingeschränkt produktive Wortbildungsmuster schwieriger zu realisieren sein dürften als solche, die massenweise produktiv sind. ${ }^{8}$ Wichtig ist dies insbesondere in Bezug auf die Sprachproduktion: Bei sehr produktiven Wortbildungsmustern bzw. -mitteln hat ein Lernender offenbar eine größere Chance, ,Treffer' zu erzielen als es bei eingeschränkt oder nur schwach produktiven der Fall ist (vgl. Storch 1979b: 4).

Schwieriger zu lernen sind seiner Meinung nach auch Wortbildungsmuster, bei denen die Wortbildung mit syntaktischen Konsequenzen verbunden ist, als solche, deren Affixe rein semantische Modifikation der Bedeutung der Basislexeme bewirken, z.B. telefonieren mit jdm. / etw. vertelefonieren und Häuschen, Städtchen. (Storch 1979b: 4)

Überdies bereiten laut Storch einem DaF-Lernenden die zahlreichen Systemlücken Probleme: „Wortbildungsmuster mit vielen sprachinternen, normbedingten Systemlücken [...] dürften schwerer sein als solche mit außersprachlich bedingten Systemlücken“ (Storch 1979b: 4). Wie soll der Lernende z.B. wissen, dass von treu, echt, böse keine Verben der Zustandsveränderung abgeleitet werden können, weder *vertreuen (treu werden) noch *echtigen (echt machen), oder dass es neben Läufer, Raucher, Fahrer nicht *Kocher oder *Bauer als Agens-Nominalisierungen von kochen bzw. bauen

${ }^{8}$ Genauer dazu siehe Storch 1979b: 4; Fleischer/Barz 1995. 
gibt (Storch 1999: 93). Mit außersprachlich bedingten Systemlücken sind solche Wortbildungen gemeint, die es nicht gibt, weil sie aus außersprachlichen Gründen nicht benötigt werden, weil das, was sie bezeichnen können, nicht existiert, z.B. Hundekrone (aber Königskrone) (vgl. Storch 1979b: 4).

Unregelmäßigkeiten bestehen weiterhin beim Umlaut, denn trotz gleicher Ablautreihe der Basisverben werden Nomen oft unterschiedlich gebildet, z.B. a-ä-ie-a: Bläser, Empfänger, aber: Anhalter, Berater; und sie sind auch bei den Fugenelementen in den Wortbildungsprodukten zu finden, z.B. Landhaus, Landsmann, Landesfarben, Länderspiel (Storch 1999: 93).

Probleme beim Lernen lassen sich zudem im Falle der Wortbildungsmuster erkennen, die keine Entsprechungen in der Muttersprache des Lernenden haben; ebenso bei den Wortbildungsmustern mit vielen Irregularitäten, die offensichtlich schwieriger sind als solche mit wenigen oder keinen Unregelmäßigkeiten, z.B. Sprung, Klang, Band, Biss (Nomina actionis bei starken Verben) und lossingen, lostanzen, losrauchen usw (Storch 1979b: 4). ${ }^{9}$

Trotz der gelisteten Schwierigkeiten darf die Wortbildung im Deutschunterricht weder außer Acht gelassen noch lediglich in geringerem Maße behandelt werden. Schon in den ersten Unterrichtsstunden tauchen die zusammengesetzten und abgeleiteten Wörter auf, wenn auch nicht in produktiven, so doch in rezeptiven Übungen zum Leseverstehen. ${ }^{10}$ Mit der Zahl der Lektionen und Unterrichtsstunden nimmt ihre Menge ständig zu, und folglich sollten sie auch im Inhalt des Unterrichts unter Beachtung bestimmter Regeln berücksichtigt und thematisiert werden. Das Regelhafte kann gelernt und geübt werden, für das Irreguläre müssen die Lernenden im produkti-

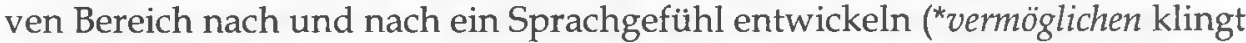
merkwürdig, befreien habe ich vielleicht schon einmal gehört) (Storch 1999: 93).

\section{WORTBILDUNGSARBEIT UND DIE DIDAKTIK DES DEUTSCHUNTERRICHTS}

Im vorliegenden Abschnitt werden die von Saxer (1991: 56ff.) und Storch (1999: 93) genannten methodischen Möglichkeiten der Wortbildungsarbeit im Unterricht entfaltet, die sich hauptsächlich aus den Merkmalen und Strukturen der Wortbildung ableiten lassen. Außer den beiden Autoren gibt

${ }^{9}$ Für die oben aufgelisteten Schwierigkeiten ließen sich vielleicht Gegenbeispiele finden, aber in den meisten Fällen treffen sie zu und zeigen dadurch eine bestimmte Tendenz auf, "[...] insbesondere wenn man sie nicht isoliert, sondern im Zusammenhang bewertet" (Storch 1979b: 4).

10 Siehe z.B. Geni@l KB1:11/11. 
es in der Literatur kaum umfassendere Vorschläge für den Umgang mit der Wortbildung im fremdsprachlichen Deutschunterricht. ${ }^{11}$

Storch (1999: 93) stellt in seinen Ausführungen zunächst die wichtigste These für die Wortbildungsarbeit im Unterricht auf, der zufolge der Bereich der Wortbildung von Anfang an systematisch in den Unterricht einbezogen werden sollte, wobei die Lernziele bei Anfängern vor allem im rezeptiven und reproduktiven Bereich liegen. Saxer (1991: 57) postuliert, dass die Lernenden auch in Wörtern, die ihnen noch nicht bekannt sind, Wortstämme wiedererkennen und durch Analyse der Wortbildungsmittel im Zusammenhang mit dem inhaltlichen bzw. situativen Kontext, in dem die Wörter stehen, deren Bedeutung wenigstens in einem groben Verständnisrahmen zu erfassen versuchen.

Storch (1999: 93) plädiert dafür, dass vor allem Wortbildungen aus stark besetzten und wichtigen Mustern in den Lehrmaterialien gehäuft vorkommen sollten, sodass die Lernenden für das Phänomen Wortbildung sensibilisiert werden und ein Gefühl für "mögliche Wörter" des Deutschen gewinnen. Außerdem ist er der Meinung, dass vor allem sehr produktive und stark besetzte Muster geübt werden sollten und weist darauf hin, dass die meisten der Wortbildungen, die der Muttersprachler oft als Fertigwort abruft und nicht mehr neu bildet, für den Fremdsprachenlerner neu sind. Das heißt, das Wortbildungspotenzial ist für den Fremdsprachenlerner wesentlich höher als für den Muttersprachler (vgl. Storch 1999: 93).

Saxer (1991: 57) hebt die Tatsache hervor, dass die Wortbildung ebenso wenig wie die Grammatik bzw. Lexik isoliert und losgelöst von den Situationen und Textinhalten, die den Ausgangspunkt für die sprachsystematische Arbeit bilden, bearbeitet werden soll, sondern immer ausgehend von den verwendeten Texten und Inhalten. Er betont, dass sich wegen der sehr vielfältigen Bedeutungsmodifizierung eines Wortes durch die Bildungsmittel aufgrund von bekannten Wortstämmen und Wortbildungsmitteln die exakten Bedeutungen neuer Wörter nicht mit ganzer Sicherheit erschließen lassen und "[...] gerade deshalb sollte man im Bereich der Wortbildung Impulse zum entdeckenden Lernen im Sinn der modernen Lesedidaktik geben als ersten Schritt des Leseverständnisses bzw. Hörverständnisses [...]" (Saxer 1991: 57). Die Lernenden sollten seinen Ausführungen zufolge auf der Basis des Wiedererkennens bzw. der Kenntnis von Wortstämmen, Wortbildungsmitteln und Bildungsprozessen im Verbund mit der Einschätzung des inhaltlichen Kontexts versuchen, einen Text global zu verstehen. Bei den Lernenden kann auf diese Art und Weise der Mut zu einem hypothetischen und fragmentarischen Verstehen von Wörtern und Texten als Vorausset-

${ }_{11}$ Siehe auch Storch 1979a, 1979b, Storch/Storch-Luche 1979. 
zung zur Herstellung des vollständigen und genauen Verstehens entwickelt werden (vgl. Saxer 1991: 58).

Ferner merkt er an, dass wenn man eine Wortbedeutung im Kontext allerdings genauer erfassen wolle, eine weiterführende lexikalische Arbeit notwendig sei. „Dabei kann man in einem Großteil der Fälle nicht wie im Bereich der Grammatik mit klaren und eindeutigen Regularitäten arbeiten [...], sondern man muss die Sprecherintention [...], den situativen Zusammenhang $[\ldots]$ oder die metaphorisch lexikalisierte Bedeutung $[\ldots]$ als Grundlage der Wortbedeutung mit berücksichtigen" (Saxer 1991: 57). Diese Vielfalt an möglichen Bedeutungszusammenhängen kann nur lexikalisch, d.h. mit Hilfe von Wörterbüchern, erschlossen und angeeignet werden, wozu die Wörterbucharbeit als Kontrolle für die Genauigkeit bzw. Richtigkeit der vermuteten Bedeutungen unerlässlich erscheint.

Wie bereits anfangs erwähnt, ist die Wortbildung ein ausgesprochen produktiver Bereich der Sprache, in dem ständig neue Lexik gebildet wird. Die Wortbildung im Unterricht kann daher laut Saxer (1991: 57f.) auch eine Spielwiese für den kreativen Umgang mit Sprache bedeuten. Er schlägt vor, dass man den Lernenden die Möglichkeit geben sollte, immer wieder die Prozesse der Wortbildung (Ableitung, Zusammensetzung) selbst produktiv im Unterricht anzuwenden, um neue Wörter zu bilden, auch auf den Verdacht hin, dass es diese Wörter im Deutschen nicht gibt. Er lenkt die Aufmerksamkeit auch darauf, dass der Sprachlehrer als Kontrollinstanz in solch einem Unterricht fungiert und die Neubildungen mit den Lernenden besprechen und ihnen erklären sollte, welche Bildungen im Deutschen gebräuchlich sind, welche nicht, welche zwar nicht gebräuchlich, aber verständlich sind und daher in bestimmten Zusammenhängen auch Verwendung finden könnten. Solcher Umgang mit der Wortbildung im Unterricht kann die Lust an der aktiven und produktiven Arbeit mit Sprache erheblich fördern.

Saxer (1991: 58) sieht noch ein spezielles Problem im Umgang mit der Wortbildung in der Lexikalisierung. Dazu gehören erstens Ableitungen und Zusammensetzungen, die sich sprachgeschichtlich früh herausgebildet haben und daher nicht mehr als solche, sondern als feste Bestandteile des Wortschatzes empfunden werden (z.B. Jungfrau); zweitens Wörter, bei denen lexikalisierte und abgeleitete Form nebeneinander stehen (z.B. verlegen: veröffentlichen / an eine andere als sonst übliche Stelle legen und deshalb nicht wieder finden). Für ihre Vermittlung muss ebenfalls Zeit im Unterricht aufgebracht werden.

Letztendlich gilt es Saxer zufolge (1991: 58) die Lernenden mit analytisch-rezeptiven Wiedererkennungsmodi wie auch produktiven bzw. spielerischen Aktionen im Bereich der Wortbildung basierend auf einer klaren 
und strukturierten Übersicht über die einzelnen Wortbildungsbereiche, gegliedert nach den Wortarten und Wortbildungsprozessen, bekannt zu machen. Er begründet diese Ansicht mit der Feststellung, dass eine entsprechende Anordnung von Informationen in qualitativer und quantitativer Hinsicht im Wortbildungsbereich den Lernenden helfen kann, sich besser auf dem Gebiet der Wortbildung im Deutschen zu orientieren und daraus für die Wortbildungsarbeit im Unterricht zu schöpfen. ${ }^{12}$

Resümierend ist zu konstatieren, dass die Umsetzung theoretischer Erkenntnisse der Wortbildungslehre im Fremdsprachenunterricht darauf abzielt, entsprechende Regeln der Wortbildung den Lernenden bewusst zu machen, was „[...] eine wichtige Voraussetzung für das Einschleifen eines "dynamischen Bildungsmusters" und für eine kreative Bewältigung künftiger Kommunikationsaufgaben [...]" (Cieszkowski 1993: 19f.) bildet.

\section{ABSCHLIEßENDE BEMERKUNGEN}

Angesichts des in diesem Beitrag angesprochenen Einsatzes der Wortbildungslehre im "Fremdsprachenunterricht, sollte dieser wichtige Gegenstand von Beginn eines Deutschkurses an - trotz potentieller Schwierigkeiten, die er in sich birgt - systematisch eingeführt werden, sei es in der Schule, im Studium oder in anderen Lernzusammenhängen. Es ist von vielen Faktoren abhängig, welche Wortbildungsmuster und -mittel sich zum jeweiligen Zeitpunkt vermitteln lassen (u.a. vom Schwierigkeitsgrad, von der quantitativen Besetzung ebenso wie vom kommunikativen Wert). Es besteht aber kein Zweifel darüber, dass je eher mit der Vermittlung von Kompetenzen bei der Wortbildung im Unterricht begonnen wird, desto selbstverständlicher und aufgeschlossener wird der Lernende ihr begegnen, bewusster mit ihr im Unterricht umgehen und sie in sein Strategierepertoire aufnehmen. Man kann sogar die Feststellung wagen, dass der Verzicht auf die Behandlung der Wortbildung im Unterricht einen Verstoß gegen die Sprachwirklichkeit bedeutet (vgl. Storch 1979b: 10). So gibt es kaum einen Text, in dem wir es nicht mit Wortbildungsprodukten zu tun haben. Außerdem entspricht der Gedanke einer Beschäftigung mit der Wortbildungsproblematik von Anfang an den heutigen Richtlinien des Fremdsprachenunterrichts. Die Vermittlung von Wortbildungsregeln fügt sich ein in die Voraussetzungen eines selbstbestimmten, autonomen Lernens, das im Zentrum des Interesses der Fremdsprachendidaktiker steht.

Zusammenfassend sei nochmals betont, dass der Wortbildungslehre beim Fremdsprachenlernen eine enorme Bedeutung zuzuschreiben ist. Der

${ }^{12}$ Genauer dazu siehe Saxer 1991: 58. 
gewünschte Effekt dieser Aufmerksamkeitsfokussierung sollte sein, dass um der Forderung von Cieszkowski (1993: 22) Nachdruck zu verleihen - die Ergebnisse einer methodisch-spezifischen Konfrontation auf dem Gebiet der Wortbildung "[...] in den Fremdsprachenunterricht und entsprechende Studienmaterialien einfließen sollen. Erst dann kann die Wortbildung systematisch vermittelt und gelehrt und nicht nur am Rande jeglicher sprachlicher Schwerpunkte im Unterricht erwähnt werden" (Cieszkowski 1993: 22). Um diesen Anforderungen gerecht zu werden, sind zukünftige umfassendere Untersuchungen unerlässlich.

\section{LITERATUR}

Bimmel, P./Rampillon, U., 2000. Lemerautonomie und Lernstrategien. Berlin u.a.: Langenscheidt. Cieszkowski, M., 1993. Zur Behandlung der Wortbildung im Fremdsprachenunterricht in Polen. In: Cieszkowski, M./Koźmin, P. (Hrsg.), Spezifika des Faches Deutsch als Fremdsprache und ihre Umsetzung in der Ausländerbildung. Bydgoszcz / Greifswald, 19-22.

Donalies, E., 2005. Die Wortbildung des Deutschen. Ein Überblick. Tübingen: Gunter Narr Verlag. Fleischer, W./Barz, I., 1995. Wortbildung der deutschen Gegenwartssprache. Tübingen: Niemeyer.

Löschmann, M., 1993. Effiziente Wortschatzarbeit. Alte und neue Wege. Frankfurt/Main: Peter Lang Verlag.

Neuner, G./Hunfeld, H., 1993. Methoden des fremdsprachlichen Unterrichts. Berlin: Langenscheidt.

Neuner-Anfindsen, S., 2005. Fremdsprachenlernen und Lernerautonomie. Baltmannsweiler: Schneider Verlag Hohengehren.

Saxer, R., 1991. Wortbildung im Sprachunterricht. Didaktische und methodische Überlegungen. In: Info DaF 18,1, 55-62.

Storch, G., 1999. Deutsch als Fremdsprache. Eine Didaktik. München: Wilhelm Flink Verlag.

Storch, G., 1979a. Wortbildung und Fremdsprachenunterricht. Teil I. In: Zielsprache Deutsch $10,2,2-13$.

Storch, G., 1979b. Wortbildung und Fremdsprachenunterricht. Teil II. In: Zielsprache Deutsch 10,3, 2-12.

Storch, G./Storch-Luche, M., 1979. Wortbildungsübungen im Fremdsprachenunterricht. In: Zielsprache Deutsch 10,4, 11-23.

Wilss, W., 1986. Wortbildungstendenzen in der deutschen Gegenwartssprache. Tübingen: Gunter Narr Verlag.

\section{LEHRWERKE}

Esterl, U./Körner, E./Einhorn, A./Kubicka, A., 2006. Team Deutsch. Podręcznik do nauki języka niemieckiego dla gimnazjum 1. Stuttgart: Ernst Klett Sprachen/Poznań: Wydawnictwo Lektor Klett.

Esterl, U./Körner, E./Einhorn, A./Jenkins, E.-M., 2006. Team Deutsch. Ksiqżka ćwiczeń do nauki jezzyka niemieckiego dla gimnazjum 1. Stuttgart: Ernst Klett Sprachen/Poznań: Wydawnictwo Lektor Klett. 
Esterl, U./Körner, E./Einhorn, A./Kubicka, A., 2007. Team Deutsch. Podręcznik do nauki jezyka niemieckiego dla gimnazjum 2. Stuttgart: Ernst Klett Sprachen/Poznań: Wydawnictwo Lektor Klett.

Esterl, U./Körner, E./Einhorn, A./Jenkins, E.-M., 2007. Team Deutsch. Ksiqżka ćwiczeń do nauki języka niemieckiego dla gimnazjum 2. Stuttgart: Ernst Klett Sprachen/Poznań: Wydawnictwo Lektor Klett.

Esterl, U./Körner, E./Einhorn, A./Kubicka, A., 2007. Team Deutsch. Podręcznik do nauki języka niemieckiego dla gimnazjum 3. Stuttgart: Ernst Klett Sprachen/Poznań: Wydawnictwo Lektor Klett.

Esterl, U./Körner, E./Einhorn, A./Kubicka, A./Jenkins, E.-M., 2007. Team Deutsch. Książka ćwiczeń do nauki jezzka niemieckiego dla gimnazjum 3. Stuttgart: Ernst Klett Sprachen/Poznań: Wydawnictwo Lektor Klett.

Funk, H./Koenig, M./Koithan, U./Scherling, T./Keller, S./Mariotta, M., 2002. Geni@l. Jezyk niemiecki dla młodzieży. Podręcznik A1. Berlin, München: Langenscheidt.

Keller, S./Mariotta, M./Scherling, T./Podkowińska-Lisowicz, M., 2002. Geni@l. Jezzyk niemiecki dla młodzieży. Cwiczenia A1. Berlin, München: Langenscheidt.

Funk, H./Koenig, M./Koithan, U./Scherling, T./Keller, S./Mariotta, M., 2003. Geni@l. Jezzyk niemiecki dla mtodzieży. Podręcznik A2. Berlin, München: Langenscheidt.

Keller, S./Mariotta, M./Scherling, T./Podkowińska-Lisowicz, M., 2003. Geni@l. Język niemiecki dla młodzieży. Ćwiczenia A2. Berlin, München: Langenscheidt.

Funk, H./Koenig, M./Koithan, U./Keller, S., 2004. Geni@l. Język niemiecki dla mtodzieży. Podręcznik B1. Berlin, München: Langenscheidt.

Keller, S./Koithan, U./Funk, H./Koenig, M., 2004. Geni@l. Jazyk niemiecki dla mtodzieży. Cwiczenia B1. Berlin, München: Langenscheidt.

Kopp, G./Frölich, K., 2001. Pingpong Neu 1. Lehrbuch. Ismaning: Max Hueber Verlag.

Kopp, G./Frölich, K./Podkowińska-Lisowicz, M., 2001. Pingpong Neu 1. Arbeitsbuch. Ismaning: Max Hueber Verlag.

Kopp, G./Frölich, K., 2001. Pingpong Neu 2. Lehrbuch. Ismaning: Max Hueber Verlag.

Kopp, G./Frölich, K./Podkowińska-Lisowicz, M., 2001. Pingpong Neu 2. Arbeitsbuch. Ismaning: Max Hueber Verlag.

Bovermann, M./Frölich, K./Georgiakaki, M./Kopp, G., 2003. Pingpong Neu 3. Lehrbuch. Ismaning: Max Hueber Verlag.

Bovermann, M./Frölich, K./Georgiakaki, M./Kopp, G./Tokmakidou, S., 2003. Pingpong Neu 3. Arbeitsbuch. Ismaning: Max Hueber Verlag. 
